\title{
THE IMPACT OF THE PARADIGM OF COMPLEXITY ON THE FOUNDATIONAL FRAMEWORKS OF BIOLOGY AND COGNITIVE SCIENCE
}

\author{
Alvaro Moreno, Kepa Ruiz-Mirazo and Xabier Barandiaran
}

\section{INTRODUCTION: TWO TRADITIONAL WAYS OF DOING SCIENCE}

According to the traditional nomological-deductive methodology of physics and chemistry [Hempel and Oppenheim, 1948], explaining a phenomenon means subsuming it under a law. Logic becomes then the glue of explanation and laws the primary explainers. Thus, the scientific study of a system would consist in the development of a logically sound model of it, once the relevant observables (state variables) are identified and the general laws governing their change (expressed as differential equations, state transition rules, maximization/minimization principles,...) are well determined, together with the initial or boundary conditions for each particular case. Often this also involves making a set of assumptions about the elementary components of the system (e.g., their structural and dynamic properties) and modes of local interaction. In this framework, predictability becomes the main goal and that is why research is carried out through the construction of accurate mathematical models. Thus, physics and chemistry have made most progress so far by focusing on systems that, either due to their intrinsic properties or to the conditions in which they are investigated, allow for very strong simplifying assumptions, under which, nevertheless, those highly idealized models of reality are deemed to be in good correspondence with reality itself.

Despite the enormous success that this methodology has had, the study of living and cognitive phenomena had to follow a very different road, because these phenomena are produced by systems whose underlying material structure and organization do not permit such crude approximations. Seen from the perspective of physics or chemistry, biological and cognitive systems are made of an enormous number of parts or elements interacting in non-linear and selective ways, which makes very difficult their tractability through mathematical models. In addition, many of those interacting elements are hierarchically organized, in a way that the "macroscopic" (observable) parts behave according to rules that cannot be, in practice, derived from simple principles at the level of their "microscopic" dynamics.

Handbook of the Philosophy of Science. Volume 10: Philosophy of Complex Systems.

Volume editor: Cliff Hooker. General editors: Dov M. Gabbay, Paul Thagard and John Woods. (C) 2009 Elsevier BV. All rights reserved. 
As a consequence, scientists have approached in a radically different way the study of these systems. Instead of looking for universally applicable laws or predictive models, biologists and cognitive scientists have searched to understand the behaviour of a living or cognitive organism by decomposing it into various parts, analysing them separately, and, then, investigating how these interrelate and affect one another within the whole system. Actually, both biologists and cognitive scientists have been able to develop rather successful research programs in their respective domains, consisting in the search for the specific material mechanisms ${ }^{1}$ that would explain the complex dynamic behaviour of those systems [Bechtel, $2006 ; 2007]$. In this mechanistic context, an explanation is viewed as the description of mappings between relevant functional operations and distinguishable structural components. And that way of conceiving and applying the Cartesian analytic-decomposition method ${ }^{2}$ has had, indeed, fruitful results: it has produced fundamental knowledge about the basic structure and - though in a lesser degree - also about the integration of many functional parts of living and cognitive systems (molecular and physiological in the first case, neuronal or abstract representational in the second one). However, this knowledge can only be considered as partial: it does not predict or fully explain the dynamic behaviour of such systems taken as complete entities, interacting in/with a variable and constraining environment; and neither does it allow building a general theory that could be applied to any system belonging to those domains of study.

Therefore, the development of science up to the $20^{\text {th }}$ century brought about two very different bodies of knowledge: in the realm where things are simple enough (though they are never as simple as they may seem), scientists were able to explain natural phenomena by means of fundamental interactions, laws of motion, basic dynamic principles and alike, generating mathematical models and theories that are predictive and can be checked through empirical techniques; whereas when the level of intricacy rises, not only in terms of the diversity of components of the system but also of their modes of organization (functional integration), the main contribution of science has been to identify and classify in a rigorous way the diverse types of material mechanisms underlying those phenomena. This, of course, included the elaboration of experimentally testable models, but with more of a justificatory or explanatory purpose than with real predictive power ${ }^{3}$.

Both ways to do science have attained an unquestionable success. However, and for different reasons, there remained many other phenomena, not only in the field of bio-cognitive sciences but also in physics and chemistry (e.g., superconductivity, complex fluid convection patterns, reaction-diffusion propagating oscillations,

\footnotetext{
${ }^{1} \mathrm{~A}$ mechanism can be defined as a structure performing a function in virtue of its components parts, component operations, and their organization [Bechtel and Abrahamsen, 2005].

${ }^{2}$ Since Descartes, the workings of life and mind (except human conscious mind) have been likened to the working of machines and physiology has been seeking to interpret the organism as a complicated set of mechanisms.

${ }^{3}$ This lack of predictive power can also be attributed to theories that have tried to explain the appearance of those mechanisms - or their highly complex design — in historical terms, like Darwin's theory of evolution.
} 
etc.), that were not amenable to any of these two traditional types of scientific approach. We are referring to what has been called "emergent" or "self-organizing" phenomena [Yates, 1987]. This type of non-linear, collective phenomenon, which was found to occur in a large variety of domains (physics, chemistry, biology, ecology, neuroscience, society, ...) shows the property that when a certain threshold in the number (and/or type) of interactions is reached, an emergent global pattern appears. Until recent times, these systems challenged scientific understanding because their behaviour could not be easily predicted, and the specific forms of those patterns were never fully explained through available mathematical models, based on fundamental laws of nature. Furthermore, the existence of a high number of variables interacting in non-linear ways precluded any possible reduction of that behaviour to a simple aggregation of the properties of the parts.

However new scientific approaches to this kind of phenomena have permitted a radical change. The advent of the sciences of complexity provided new tools and modelling techniques (such as cellular automata, genetic algorithms, Boolean networks, chaos and dynamical systems theory) to really tackle scientifically some of those problematic phenomena, and challenged, indeed, the simplified picture of science portrayed above. Interdisciplinary research combined with the increasing power of computer simulations made possible an unprecedented progress in our knowledge and comprehension of these systems. It is the concept of network and its mathematical and computational expressions that have provided the most fruitful metaphor and model for non-decomposable complex systems. We shall call 'network theory' the set of mathematical and computer simulation models and tools that have being developed to study network architectures and dynamics. Although there is no unified branch or corpus of mathematics that constitutes network theory, there exists however an increasingly indispensable 'tool-kit' of methods and disciplines that merge into what we might call network theory: this ranges from dynamical systems theory to network topology, from random boolean network models to coupled oscillators (see [Strogatz, 2001] for a survey). The study of networks with strongly and recurrently interacting components allowed scientists to deal with holistic systems, showing that, despite their variety, they share certain generic properties. For many, these advances seemed to reflect some common, universal architecture of complex systems (or, at least, the fact that they all belonged to a critical region 'at the edge of chaos' - [Langton, 1990; Lewin, 1992]).

So the blossoming of the sciences of complexity, among other important reasons, induced -and is still inducing- a profound change in biology and neuroscience toward less analytic and more synthetic-holistic views. For many, what these advances have shown is that the time is coming for converting biology and cognitive science into "true scientific disciplines", following the model of physical sciences. However, as we discuss below, it is still unclear whether the common features found through these network studies (e.g.: edge of chaos, self-organized criticality, power-law distributions, small worlds, etc...) actually respond to similar or completely different underlying, generative mechanisms. Indeed, when we 
examine more carefully biological and cognitive systems, a much wider variety of interacting components and non-linear interaction modes are found (as compared with any physical or chemical complex system). More precisely, biological and cognitive systems show an intricate and rich functional diversity within a modular and hierarchical dynamic organization. This organization is endowed with global/collective properties, like highly robust self-maintenance, and shows singular patterns of behaviour in their environments, like agency, multiscale adaptive flexibility, etc. Most of these features are missing from the complex dynamic models of the standard sciences of complexity, and suggests that biological and cognitive systems hide not only more complexity than physical systems, but rather different forms of it.

Acknowledging that new approaches and methodological tools are required to account for the specific types of organized complexity that generate biological or cognitive phenomena, we will discuss in what sense the 'paradigm of complexity' is deeply reframing our ways of conceiving science and philosophy of science itself. Our contribution is structured as follows: first, we explain more thoroughly how the challenge of complexity (i.e., the challenge of understanding scientifically holistic organizations) meant a very important step forward with regard to the traditional ways of doing biology or cognitive science. Then we explore how far did the 'complexity paradigm' actually go in terms of modelling biological and cognitive systems, highlighting the specificities of the latter and analysing the steps that need to be taken to capture them. And, finally, we recapitulate on the role that complexity sciences have played in the opening of what will probably constitute a new scientific era, in which up to now almost exclusively descriptive-explanatory disciplines, like biology and cognitive science, will turn into much more predictive and technologically fruitful parts of human intellectual endeavour.

\section{THE CHALLENGE OF COMPLEXITY: UNDERSTANDING HOLISTIC ORGANIZATIONS}

Seen from the modern perspective of complex systems, it is somewhat paradoxical that highly complex systems like biological and cognitive entities have been studied, for centuries, and rather successfully (at least in specific phenomenological domains), through atomistic and reductionistic methodologies, within the classical paradigm of mechanistic explanations. The mechanistic strategy assumes that the system under study is decomposable or 'nearly-decomposable' [Simon, 1962/1969]: i.e., that interactions between subsystem components do not change their structure and dynamics, so that the decomposition of the system in those sub-parts and their isolated study does not seriously threaten their stability and characteristic functioning when put back together. According to this working hypothesis (later we will explain why it can be adequate at certain levels of description), even when a system has a high number and a high diversity of parts and functions, the process of achieving a satisfactory mechanistic explanation of it does not require a change of methodological principles (although this might turn to be long and 
costly). ${ }^{4}$

Most researchers in both biology and cognitive science were probably aware that this methodology would not be able to provide models for predicting or reproducing the full, integrated behaviour of their objects of study, nor to provide a complete universal theory of them, beyond particular explanatory accounts. But it was assumed that this (if ever possible) had to come at a later stage. First of all one had to investigate whether a one-to-one mapping between structural and functional units in the system could be drawn and only later worry about more complicated relationships, or about how those units could be integrated. And here is where the 'organism-machine metaphor' came to play such an important conceptual role, as a second central working assumption, with great consequences. There were, to be sure, researchers ([Rachevsky, 1938; Bertalanffy, 1952; Elsasser, 1966] just to mention a few) that did not accept such naïve linear interpretations and reminded the scientific community of the necessity to adopt holistic, integral approaches in scientific strategies to deal with living or cognitive systems. But their actual impact until the end of the past century was very small, marginal at most.

Nevertheless, as knowledge on biological and cognitive systems became more detailed and fine-grained (i.e., as increasingly accurate biochemical and neurological understanding was gained at the level of basic constituents and elementary functions, thanks to the great advances in fields like molecular biology and neuroscience — see, e.g., [Morange, 2003; Bechtel, 2006; 2007]) researchers became progressively aware that components were acting in strongly holistic ways. For example, at the end of the last century the reductionist program based on molecular biology that was centred in the discovery of the structure of the genome faced a dead end, since the more details were known, the more evidence was accumulated that genetic components acted in a complex web of interactions. Thus, instead of focusing on the detection of genes, researchers started to look for the structure of regulatory networks at different levels (genomics, proteomics....). In other words, as E. Fox Keller has pointed out [2005a], research in biology changed from a program inscribed in DNA analysis to a new distributed (namely, more holistic) program in which DNA, RNA and protein components operate alternatively as instructions and data.

This shift has not only been a consequence of the internal development of biol-

\footnotetext{
${ }^{4}$ What the strategy of decomposition and localization permits is to isolate parts and to study them separately, in terms of their functioning, so that an adequate understanding of the system can be attained. This method has led to very important progress in the understanding of the functioning of biological and cognitive systems (e.g., early understanding of metabolic pathways, reflex arcs and circulatory system). Atomistic reductionism can be understood as a class of mechanistic explanation where a one-to-one mapping between structure and function is established and where there is just a linear aggregation of localized functions, so that the properties of the whole can be reduced to or identified with the properties of the parts. What is meant by linear aggregation (or sum) is a sequence of operations (performed by well identifiable parts). Many human-made mechanisms operate in that way: a lever moves a gear that in turn makes another gear rotate which in turn displaces a latch and the door opens. But some natural mechanisms may also be described and modeled in a similar vein: a reflex arc, the snap trap of carnivore plants, etc.
} 
ogy and cognitive science as research disciplines, but also of the already mentioned development of the sciences of complexity, which showed that the understanding of holistic systems could be closer than ever by means of strict scientific tools, especially through the application of new approaches to differential calculus (e.g., deterministic chaos, bifurcation theory, self-organized criticality) and network modelling tools that make use of computer algorithms and simulations [Waldrop, 1992; Lewin, 1992; Kauffman, 1993; Gell-Mann, 1994]. Of course, many researchers have kept working within the reductionist paradigm, which was - and will still continue to be - the central pillar of biological and cognitive sciences. But they have been, so to speak, 'filling in details'. And even if these details turned out to be very important (e.g., for medical or biotechnological applications), it does not seem that major breakthroughs in basic knowledge will come from there any more. What is still really missing, as announced above, is the combination, the integration of all that fine-grained accumulated knowledge, so that the principles of organization of those complex systems came to the surface. In other words, more global, holistic, approaches have to find their way through. Therefore, even if mechanistic explanations have not been abandoned, further progress demands that they be profoundly re-interpreted in systemic-organizational terms, as some authors have claimed in fields like cellular or neural physiology [Harold, 2001; Bechtel, 2006; 2007].

Nevertheless, the phenomenon of holistic organization is broader than biological, cognitive and social systems. Even relatively "simple" physical or chemical systems may show holistic properties. Within the classical paradigm of physical sciences the fact that there are special systems, whose properties cannot be statistically inferred, has been acknowledged since long ago [Weaver, 1948; Schrödinger, 1944]. Unlike gases and certain fluids and solids (traditional targets of physical sciences), where the global result of interacting parts can be averaged out, in these systems the non-linear and recurrent nature of the interactions between their components generates long-range correlations and global properties/patterns of dynamic behaviour, within and in relation with the environment, that hinder any straight-forward connection between levels (micro-macro, individual-collective, ... — for a more detailed account see [Collier, Hooker, 1999]). These systems, which cannot be explained by means of the usual mathematical techniques and simplifying approximations in physics, are said to be holistic because they show global emergent properties that cannot be attributed to specific or well-distinguishable parts. And the phenomenon is emergent because the linear aggregation of those parts to understand the functioning of the system as a whole is not possible: 'the whole is more than the sum of the parts' as the main slogan of the sciences of complexity runs. According to Melanie Mitchell: "Roughly, the notion of emergence refers to the fact that the system's global behaviour is not only complex but arises from the collective actions of the simple components, and that the mapping from individual actions to collective behaviour is non-trivial" [Mitchell, 2006, p. 1195].

But, interestingly, the real cause of holism goes beyond the high number of components and the non-linearity of their local interactions. The system as an in- 
tegrated whole determines in an important way how the parts behave. So, where does this "systemic causal power" come from? Instead of just an asymmetric, bottom-up, causal action from the constitutive components of the system to its global properties, a new type of process appears (in a reverse or opposite direction): the constraining action of supramolecular aggregates and long-range patterns on molecular dynamics, modifying or channelling low level interactions, so that a kind of feedback is created and a recurrent loop is eventually reached. That is why functional decomposition is not always possible or, more precisely, certain relevant properties of the system cannot really be reduced to an aggregation of its component parts. In those systems where we find holistic properties, the maintenance of the global stability is the result of a dynamical causal loop, such that, at least one macroscopic constraint (created by the underlying microscopic interactions) is causally necessary for the maintenance of such a loop. In other words, there is, in a minimal sense, a form of self-maintenance. ${ }^{5}$ It is this causal circularity that makes it impossible for any specific participation in the global result to be localized in a particular component or set of components. Therefore, complex phenomena become extremely difficult — if not impossible — to trace through purely analytic mathematical models and decompositional mechanistic strategies.

So this was a challenge for traditional physical sciences, since emergent holism is not excluded from the bare physico-chemical world. The classical examples are the so-called 'dissipative structures' [Nicolis and Prigogine, 1977], like Bénard convection cells or the B-Z reactions. But there are other similar examples in nature, like tornados, or fire flames, in which the maintenance/stability of the global pattern of order requires a dynamical 'micro-macro' causal loop. In these cases, even if some structural decomposition of the system can be achieved, the mapping between structure and function is highly problematic because there is no one-to-one correlation between structure and function. In terms of the relationship between structure and function, there is a many-to-one mapping (many components perform a single collective function, or contribute to a single pattern) that cannot be accounted for by standard statistical approaches. From this situation, things can get even more intricate if: a) the emergent pattern may change (between different macroscopic configurations) depending on internal or environmental conditions $^{6}$; b) intermediate-level patterns start to form; or, c) global self-organization

\footnotetext{
${ }^{5}$ As Van Gulick (1993) has pointed out, most patterns in the world exist because they are self-maintaining; namely, these patterns are the result of circular processes: emerging organized structures exerting selective action on the lower level dynamics of their constitutive parts, which recursively contribute to the maintenance and preservation of those very organized systems.

${ }^{6}$ Feed-back loops occur not only among components of the system but also between the system and its environment. So much so that some characteristic behaviour of the system could be impossible to study if we consider it isolated from its environment. In those cases, causal attribution of observed behaviour to internal components is, obviously, not possible. It is always important to remember this other, not less relevant aspect of complexity, coming from the interactive emergence of some functional properties in a system. In fact, this is one of the major theoretical achievements that situated robotics has contributed to in the foundations of cognitive science, showing how complex adaptive behaviour could be the result of relatively simple -yet strongly interactive- control systems in robots (Brooks 1991, Steels 1991, Clark 1997), whereas
} 
is combined with local self-assembly or self-re-structuring processes, leading to new identifiable structural/functional components in the system. In these cases, the many-to-one mapping would become many-to-many, but we will address this a bit further on, when we deal with the specific complexity found in living and cognitive systems.

As already mentioned, the study of the emergent behaviour of holistic systems has been made possible thanks to the development of powerful computing models and machines. Although the dynamic processes leading to emergent behaviours are not analytically tractable, numerical methods, consisting in a fine-grained stepby-step update and recording of the state of all the interrelated variables of the system, allows the drawing of the state space of these systems. In this way, the evolution of the system is "synthetically" reproduced in the course of the simulation, rather than deduced for a given time value. Under these conditions, the prediction of the global property requires an enormous amount of parallel computation, where the complexity of the computational simulation is almost equivalent to that of the simulated system ${ }^{7}$. Since sensitivity to initial conditions might be critical, the process is repeated for a wide range of initial conditions and the behaviour of the system recorded. A full record of different simulations allows drawing the state space of the system (given certain boundary conditions and specific parameters) so that regular and reproducible patterns of behaviour can be observed. Under different network configuration parameters, the rules governing components or boundary conditions can be systematically studied through simulation, and a deeper understanding of the structure-function mapping is gained without a strict decomposition or a one-to-one localization.

Following this methodology, different tools and models have been developed to discover generic properties of complex systems, often without a specific target system to fit and correspond with [Barandiaran and Moreno, 2006a]. These models and tools are typically used to illustrate emergent properties, to discover universal patterns of connectivity in networks and their properties. Among the early models we should mention those that focused on the special dynamic behaviour of systems in a region in between 'order and chaos', for which, indeed, the standard statistical analysis did not work. The paradigmatic example is 'spin-glasses', which led to the development of 'Ising-type' of models (originally applied to ferromagnetic systems). And, essentially, the new approach was to represent the system as a discrete set of nodes, with very limited dynamic states (typically just two: 'on/off' or 'up/down') and variable connections among them, which affected in different ways their respective states. Through this basic strategy, one could deal with systems of

previous functionalist models required a high computational load to achieve the same behavioural performance.

${ }^{7}$ According to Kolmogorov and Chaitin, if the description of the structure cannot be shortened, it is said to be (maximally) complex (see, e.g.: Li \& Vitanyi 1997). However, there are also other criteria to measure complexity, like computational time or the difficulty of finding that compressed description. For instance, a fractal structure, according to these other criteria, would still be regarded as complex, whereas from the point of view of the mathematical algorithm that generates it, it would be very simple. 
many components in a radically different way (as compared to previous statistical methods): namely, putting the emphasis on the different types/degrees of connectivity or interaction that may be established among components of a system and the effect that this has on its global properties [Weisbuch, 1986].

Actually, the classical works by Kauffman on complex gene regulation patterns (using Boolean networks) and autocatalytic sets (for a review, see [Kauffman, 1993], or Cellular Automata [Wolfram, 1986] were based on this key modelling idea. The assumption that simple local rules among discrete, randomly connected elements could give rise to unexpected global behaviours was very fruitful, leading to many other simulation algorithms and models, which were applied to study phenomena at very different scales. Quite interestingly, all those phenomena were characterized by a critical transition point (most often related to a threshold of connectivity in the network - e.g., Langton's [1990] Lamda parameter) above which global properties emerged by some sort of 'percolation' effect. The success of these pioneer models triggered increasing interest in the properties of complex networks [Strogatz, 2001] and how these could capture essential features of systems as diverse as metabolic regulation pathways [Wagner and Fell, 2001; Ravasz, et al., 2002], ecosystems [Montoya and Solé, 2002], brain networks [Sporns, et al., 2000; Sporns, et al. 2005] or the world wide web [Barabasi, Albert, 1999], which were soon discovered to share some general topological properties (e.g., power-law distributions, scale-freeness, 'small worlds', etc.).

Thus, by the end of the century a whole new scientific research program was initiated and seemed to be capable of grasping, with tools based on the increasing power of computers, (at least certain types of) prototypic holistic systems. And not only grasping but also predicting/reproducing their dynamic behaviour, as well as quantifying to a good extent their degree of complexity. A possible interpretation of all this success is that, behind the apparent complexity and variety of those systems, there are some general principles (applicable to systems with a great number of interactions, or with particular patterns of connectivity) that reflect a common architecture emerging at various phenomenological levels. ${ }^{8}$

\section{THE IMPACT OF NETWORK THINKING ON THE CONCEPTUAL CORE OF BIOLOGICAL AND COGNITIVE SCIENCES}

Living — and, thereby, cognitive — systems necessarily originated and persisted on the basis of previous physico-chemical modes of complexity. In the same way as self-organizing reaction-diffusion patterns [Turing, 1952] appear in many biochemical processes of uni- and multi-cellular organisms, the type of holistic phe-

\footnotetext{
${ }^{8} \mathrm{~A}$ more critical view on these network modelling approaches (see, e.g.: [Fox Keller 2005b]) tends not to consider their results so surprising or significant, after realizing that there are many ways, many mechanisms through which those general features can be generated in nature (and in the computer), and that the relevant information to understand complexity is contained precisely in those underlying mechanisms. We will see that this is quite true, when we examine, in the next section, the specific nature of biological and cognitive complex systems.
} 
nomena we just described (let us call it 'primary holism') is ubiquitous in the biological and cognitive world: e.g., in the cell cycle, in the symmetry breaking phenomenon related to the appearance of morphogenetic fields in development, in the 'collective intelligence' of ant or bee colonies, in the central pattern generator of neural circuits, in the chaotic dynamics of the olfactory bulb neurons in rabbits... It basically reflects how an ensemble of interacting units produces a global property or pattern of behaviour that cannot be ascribed to any single of them, but to the whole ensemble. And this is both relevant for the biological and the cognitive spheres, revealing that one-to-one mappings between structural and functional parts are typically not possible. This new vision led to a number of revisions, acknowledging the power of collective, distributed organizations: the queen did not have to instruct the ants for them to operate in a coordinated way; cells did not have to contain highly explicit genetic instructions since they could form complex dynamic patterns and tissues just following local context-sensitive rules; neurons did not have to compute symbolic propositional states but could undergo distributed sub-symbolic processing; etc.

Before the advent of network theory and the new insights it brought about, the foundations of biological and cognitive sciences had to be based on those assumptions that could meet the demands of the mechanistic-decompositional methodology. These assumptions had enormous consequences on the way cognitive and biological systems were conceptualized, particularly at higher level theories (such as principles of biological evolution or philosophy of mind). The one-to-one mapping assumption, for instance, somehow forces a jump over many levels of complexity to establish a workable one-to-one representational relationships at different scales.

In biological systems this atomistic representationalism took the form of a oneto-one genotype-phenotype mapping whereby organisms were conceptualized as phenotypes encoded on genetic strings and evolution as a process of genetic variation and retention due to a selective force that propagated linearly from phenotypes to selfish genes [Dawkins, 1974]. Research on genetic regulatory networks [Lewis, 1992] has completely transformed the foundational framework of evolutionary biology together with the study of self-organization in developmental processes [Goodwin, et al., 1983; Kauffman, 1993]: genes are now one among all the components and processes that produce organisms, within a network of internal and interactive dynamics that constitutes their life-cycle, and not a freely re-combinable encoding of biological forms. As a result, evolution does not operate upon an abstract functional phenotypic space but through a highly constrained (occasionally discontinuous) space of possible morphologies [Alberch, 1982], whose formation requires acknowledging the environmental, material, self-organized and often random processes that appear networked at different scales. Another assumption that was strongly revised was the sharp system-environment distinction, which was made necessary in order to map internal structures to environmental conditions. Thus, now it is not the environment that poses problems for genetically instructed phenotypes to solve, but a complex organism-environment interaction process that defines the adaptation and survival of an organism [Lewontin, 2000], including 
ecological and niche-construction processes [Laland, et al., 1999].

The number of transformations that the sciences of complexity made in the foundations of biology was comparable to those made in cognitive science. Symbolic computational units and their rule based manipulation were postulated as theoretical primitives by functionalist approaches to the mind [Fodor, 1975; Newell, 1980; Block, 1980]. Cognition was primarily conceptualized as a set of inner symbol manipulation procedures on the side of the subject, mirroring external states of affairs (and a one-to-one mapping between internal abstract functions and environmental structures) in order to deliver planned actions to be executed in the world. The rise of artificial neural networks and parallel distributed processing in the 80's started to undermine the atomistic symbolic assumption leading to a subsymbolic processing paradigm where cognitive architectures became biologically grounded on the distributed nature of brain architectures [Rumelhart, et al., 1986; Hopfield, 1982]. During the 90 's the subject-object dichotomy came to be challenged by situated robotics, whose engineering principles exploited recurrent system-environment interactions to generate emergent functional behaviour [Brooks, 1991; Steels, 1991]. Around the same time, dynamical system approaches to cognition [Kelso, 1995; van Gelder and Port, 1995] started to conceptualize cognition as a dynamical process where cognitive behaviour and development emerged from dynamically coupled brain-body-environment systems [Beer, 1995; Thelen and Smith, 1994] challenging previous assumptions about the innateness of cognitive capacities [Elman, et al., 1996] and their purely internalist and disembodied computational bases. Where previous mainstream neuroscience was mostly focused on finding neural correlates of cognitive representations and localizing functions, brain activity started to be understood as emerging from the collective dynamics of distributed neural ensembles at the edge of chaos [Skarda and Freeman, 1987; Tsuda, 2001] integrating its biological embodiment as a complex interplay between internal bioregulatory functions and sensorimotor activity [Damasio, 1994; Lewis, 2005]. Thus the clear cut subject-object dichotomy and the abstract atomistic computational framework came to be progressively substituted by an internally and interactively distributed network of dynamical processes capable of giving rise to context-sensitive, flexible and multiscale adaptive capacities, equally constrained and enabled by its biological embodiment.

Despite the early warnings about the consequences that reductionist approaches could have for conceptualizing biological and cognitive systems [Waddington, 1957; Bertalanffy, 1952; Merleau-Ponty, 1963], only when the network theory toolkit became available to explore the intricate and emergent structure-function mappings in metabolic, neural, ecological, developmental, behavioural and other types of phenomena, did the true complexity of life and cognition begin to be fully acknowledged and the old foundational framework progressively transformed, substituting its traditional simplifying assumptions by more encompassing and realistic ones. 


\section{SPECIFICITIES OF BIOLOGICAL AND COGNITIVE COMPLEXITY}

Therefore, we can say that complexity sciences have already contributed in a significant way to a better understanding of living and cognitive systems, through the new insights and tools provided to deal with that primary or most elementary form of holism, as described in the previous section. However, the complexity of these systems goes far beyond there. It involves a much richer internal structure, with functional diversity integrated in second-order forms of holism. Biological and cognitive systems are certainly made of a large number of parts or elements acting in non-linear ways, but they also show other features that are absent in non-living complex systems: hierarchical organization, long-term sustainability, historicity, functional diversity, adaptivity and agency. Everywhere in biology and in cognitive science we deal with systems made of parts or elements with different functionalities acting in a selective and harmonized way, coordinating themselves at different time scales, interacting hierarchically in local networks, which form, in turn, global networks and, then, meta-networks... The organization of the living systems consists in different nested and interconnected levels which, being somewhat self-organized in their local dynamics, depend globally one upon the others. This means that both the components and the sub-networks contribute to the existence, maintenance and propagation of the global organizations to which they belong. And, in turn, those global organizations contribute to the production, maintenance and propagation of (at least some of) their constitutive components. ${ }^{9}$

Thus, this complexity is quite different from what we can find in non-living systems, like the dynamical complexity of holistic non-linear networks in both physical and chemical domains. It is a type of complexity that goes beyond a mere increase in the "complicatedness" (i.e., an increase in the number and variety of components) of self-organizing systems: it involves qualitative changes in the form of organization of the system, by means of creating functionally differentiated structures and levels within it. So here we are dealing not with mere complexity but with organized complexity as Weaver [1948] called it. Nevertheless, by using this term we mean something more than Weaver's idea of systems consisting of many different elements interrelated into an organic whole; rather, we want to refer to the fact that these systems regulate and functionally manage their own complexity. As J. Mattick [2004] has recently pointed out, what really matters in biological evolution is not so much the generation of complexity, but its functional and selective control. Evolution shows that the appearance of increasingly complex systems goes together with the invention of different ways of utilising hierarchical regulation to manage internal functional variety.

Actually, what distinguishes biological and cognitive complexity from other forms of complexity or complicatedness lies in the role played by mechanisms

\footnotetext{
${ }^{9}$ Recurrent-functional relationships established between the parts and the whole of a system expand to interactions between the system and its environment. The latter are controlled by the system, so that they contribute to the system maintenance. Such a control is modulated by the internal and external (adaptability) conditions and constitutes the so-called agent's behaviour.
} 
of regulatory control in the functioning of these systems [Christensen, 2007]. By the term "regulatory control" we mean an action performed by a certain part (component or substructure) of an organized system in a way that some processes occurring in it are functionally constrained: in other words, their dynamics is partially harnessed, or channelled in order to ensure or improve the maintenance of the system as a whole. For example, a metabolism is a self-maintaining organization that functionally modulates its ways of operating when perturbations occur (e.g., concentrations of internal metabolites are maintained despite changes in some of the inputs/outputs), whereas a whirl is a non-regulated self-maintaining organization (since a totally distributed holistic network, although it may be more or less robust, is not capable of self-regulation). Equally, neuronal and interactive selforganization may generate some interesting behavioural patterns; however, some means of action selection would also be required if the agent is to organize a complex behavioural repertoire according to a given set of priorities [Prescott, 2007]. Some early pioneers of the paradigm of situated and autonomous robotics (which was inspired, to a certain degree, in the sciences of complexity — particularly in the ideas of self-organization), after an initially celebrated success, soon became aware that self-organization alone faced a bottleneck of complexity growth and that modular and hierarchical control principles had to be reintroduced in order to scale up [Nolfi, 1997; Brooks, 1997].

This highly organized complexity cannot be the result of the "one-shot, orderfor-free, kind of self-organization associated with the kinds of uniform non-linear dynamical systems that mathematicians usually study", as E. Fox Keller [2007, p. 316] has pointed out. Rather, it requires an organization structured in different (sub)systems interconnected at different levels and time scales. ${ }^{10}$ In other words, it requires the creation, within the system, of dynamically decoupled ${ }^{11}$ subsystems that can modify the parameters of other subsystem parts. Since these subsystems work at different rates and with different operational rules, the system has an

\footnotetext{
${ }^{0}$ Organisms are internally organized in different networks and sub-networks of dynamicalmetabolic processes. But, in addition, they are embedded in a more global evolutionary metanetwork, articulated also on different levels and functioning at much wider spatial and temporal scales. Slow, phylogenetic processes are causally connected with faster, ontogenetic ones, generating a global long-term sequence of spiral-like cycles, that we call "evolution", which allows cumulative increases in complexity. The long-term sustainability of this complexity is based on the spatial and temporal interconnection and interdependence of the individual organisms, generating a wide meta-system (an eco-system or, even, a whole biosphere). An essential characteristic of these higher forms of complexity is, thus, their inclusion in a long-term evolutionary phenomenon. Life started as some minimal form of organization (population of prokaryotes) and developed in a continuous and causally interconnected way. As a consequence, historicity is also a fundamental component of biological and cognitive complexity.

${ }^{11}$ By the term "dynamical decoupling" [Moreno and Lasa, 2003; Ruiz-Mirazo et al., 2007] we are referring to the organization of a system such that the basic, constitutive processes generate and sustain a relatively independent dynamic subsystem, which in turn acts selectively regulating those constitutive processes. This means that there are, at least, two organizational levels (although in highly complex biological systems there are many more): the constitutive system is the basic or "lower" level and the regulatory subsystem is the "higher" one. Significantly, the higher-level regulatory subsystem cannot exist without the regulated constitutive system: both levels causally depend each other, and therefore the whole system appears as an integrated unity.
} 
increased potential to explore new or alternative forms of global self-maintenance (that are not accessible to 'flat' systems without any hierarchy or modularity in their organization). In this way, the higher level subsystem creates a set of functional constraints on the lower-level dynamics. At the same time, the controlled level plays a fundamental role in the constitution and maintenance of the controller level (and therefore, of the whole system). For example, the genetic (sub)system in the cell acts - through the synthesis of specific enzymes - as a mechanism of control, harnessing the dynamics of the metabolic reactions, but in turn, metabolic processes contribute to the maintenance, reparation, replication and translation of genetic components. Another example is the nervous system, which controls metabolic processes (circulation, digestion, breathing, etc.) on the one hand but, on the other hand, is fabricated and maintained by the latter.

Therefore, biological and cognitive systems convey specific forms of complexity that, through holistic-emergent processes (which are continuously taking place), produce both dissipative patterns and new, more complex structures which, in turn, are bound to become selective functional constraints acting on the dynamic processes that underlie those holistic processes. The reason why those functional constraints can be described as mechanisms is that they act as distinguishable parts (or collections of parts) related to particular tasks (e.g., catalytic regulation) performed in the system. So both aspects are, thus, complementary: the holism of the global network of processes and the local control devices/actions that are required for the system to increase in complexity. Moreover, the newly created and functionally diverse constraints may give rise (once a certain degree of variety is reached) to new self-organizing holistic processes, which, in turn, may be functionally re-organized. In this way, an increase in organizational complexity can take the paradoxical form of an apparent "simplification" of the underlying complicatedness, giving rise to levels of organization in which a mechanistic decompositional strategy might be locally applicable. The idea, taken originally from Pattee [1973], would be that new hierarchical levels are created through a functional loss of details of the previous ones.

However, the key point here is that this complementarity between functional mechanisms and holism, is due to their causal circularity. Since a mechanism is an explanation of the functioning of a system in terms of a specific arrangement of parts, it always sends one back to another mechanism to explain that arrangement of parts, and so on indefinitely. Thus, causal circularity is the only possible solution to the infinite regress posed by mechanistic explanations. And here is where the main difference between what we mean by a mechanistic explanation in a man-made system and in a natural one lies. Actually, the organism-machine analogy brings biological and cognitive sciences closer to engineering than to physics or chemistry, as Polanyi [1968] sharply highlighted, arguing that both (organisms and machines) operate according to local rules (or 'boundary conditions') irreducible to general physico-chemical laws/principles. ${ }^{12}$ But, whereas in man-made organiza-

\footnotetext{
${ }^{12}$ In fact, engineers care about the design of a device that operates as efficiently as possible in order to achieve an external, human-defined, purpose; but they ignore the question of how such
} 
tions structure and function are causally asymmetric (a given structure generates a given function, though not conversely) in biological and cognitive systems both structure and functions are engaged in a circular causal relation (as other authors have already claimed - [Rosen, 1991]).Since what artificial machines do (i.e., their function) does not feed back to their own structure, machines must be externally designed and repaired.

In contrast to artificial machines, in biological (and cognitive) systems the organization is internally generated, so the structure is itself the cause and the result of their functions. In other words, biological and cognitive systems are organized so that for each required operation, there is a part (or set of interconnected parts) that is apt to perform it. For instance, the main emergent property of a living cell is its maintenance through the continuous renewal of its constitutive parts and through the management of the flows of matter and energy with its environment. Highly complicated components, like enzymes, accomplish specific functions, which, in turn, ultimately regenerate these enzymes from more simple building blocks. If we compare a living cell with a computer, we can see that, although in both cases there is a highly complex internal structure, the computer functioning does not contribute to the maintenance of its own structure, whereas the cellular functioning is the cause of its structure.

Quite interestingly, this property of causal closure in 'soft material automata' (as opposed to the rigid or fixed structure of relationships in traditional man-made machines) involves high rates of energy dissipation, so it requires the continuous production of work by the system [Ganti, 1987]. Thus, living systems, which are continuously and literally fabricating themselves, can only maintain their organization in far from equilibrium conditions by being material-thermodynamically open. Nevertheless, biological and cognitive systems are something more than selfmaintaining organizations operating under specific matter-energy flow conditions. Rather, they recruit their own internal organization to actively create and maintain the internal and boundary conditions necessary for their own constitution: in other words, they are autonomous systems [Etxeberria, et al., 2000; Ruiz-Mirazo and Moreno, 2004; Barandiaran and Moreno, 2006b; Barandiaran and Ruiz-Mirazo, 2008]. As a consequence, biological and cognitive systems are not only intrinsically functional, but normatively functional systems: they not only function in a certain complex way, but they must function in that way in order to ensure their continuing existence. The key notion here is that the structure and stability of living systems are not independent of their functions but, on the contrary, that the functional level feeds-back to the structural one. There is, thus, a circular co-dependence between the stability or self-maintenance of structures and their functions.

Now, what all this makes clear is that the development of models trying to capture (and potentially reproduce) the huge complexity of biological and cognitive systems cannot be achieved without a deeper understanding of the constraints or mechanisms that allow the functional reorganization of emergent collective pat-

design could have appeared without another design (or without an ultimate human designer). 
terns: in other words, how to convert these patterns into new functional substructures in the system; and how, within a global causal circularity, these new functional substructures exert top-down regulatory actions on other components of the system. Among other things, a deeper understanding of how a self-organizing system can re-use some sets of its initial components to achieve new functions, through a 'tinkering-like' process. But we will discuss this in the next section.

\section{THE PRESENT OUTLOOK: FURTHER STEPS IN THE MODELLING OF BIOLOGICAL AND COGNITIVE SYSTEMS}

So how could the specific features of biological and cognitive systems, in particular the emergence of new functional variables and their integration in complete operational units (i.e., the actual living/cognitive organisms), be modelled? The sciences of complexity should continue giving us new insights and tools to tackle this problem, through further advances in network theory and, particularly, the development of bottom-up simulation approaches. Up to now they have already provided, apart from a solid theoretical framework to explain first-order holistic systems (as remarked above), a new way to look into the organizational sub-structure of real living and cognitive systems. This mainly derives from the extensive use that is being made of network theory to try to extract relevant information from the huge data banks provided by research in cell and developmental biology, ecology and neuroscience.

Two main messages can be drawn from their survey, so far. The first is that modularity and cross-level processes play a very important role in the organization of these highly complex systems (see, e.g., [Hartwell, et al., 1999; Solé, et al., 2002a; $2002 \mathrm{~b}]$ ). In other words, that functional decomposition is justified but has to be carried out carefully, taking into account different levels of description and their possible interconnections. The second is that redundancy and degeneracy [Tononi, et al., 1999; Edelman and Gally, 2001] are surely present at these different levels, so accurate decomposition will actually be very hard (if ever possible) to achieve. That is to say: in reality things are not as neat as they could be, since these systems appear to be the result of a long process of tinkering (not of a beautiful design made by an external, analytically inspired engineer). Indeed, nature builds up from what is already available: the metaphor of the tinker is quite appropriate [Jacob, 1977], and particularly well suited to describe how things have come together and evolved in the realm of complexity [Solé, et al., 2002b].

This general picture explains why the analytic-mechanistic approach to living and cognitive systems has been rather successful so far: despite the complexity involved, the high degree of modularity allowed for decomposition and the search for one-to-one mappings between structural and functional components. But it also sets its limits: this type of Cartesian approach can only be, in the best case (i.e., if close enough to the 'near-decomposability' condition), a first approximation to the problem. Degeneracy and redundancy - without going into the technicalities of network theory - reflect the fact that many structural components in these 
systems may actually have the same function, or that a single component may have more than one function, so the one-to-one assumption, in general, does not hold. Real complexity out there, in the biological and cognitive worlds, is not only many-to-one but certainly 'many-to-many'. Or, rather, 'many-to-many-to-all', because the presence of hierarchical, inter-level relationships indicates that the problem of integrating all these modules in a second-order holistic organization cannot be circumvented. However, this is far from being a trivial problem.

Recent advances in network approaches are pointing at this, although they are not quite there yet. Researchers in the field are moving from the current stage, in which the focus has been on the topological/architectural features of networks - like the 'small worlds' found in metabolic networks [Wagner and Fell, 2001] or in food-webs [Montoya and Solé, 2002], as mentioned before - towards reintroducing true dynamics in their graph analysis and enhancing, in this way, the bottom-up explanatory potential of the resulting models. So it is clear that the key question is not just specifying the general rules for network growth or development (e.g., preferential attachment, duplication/rewiring, optimization, ... ), but including in their models real dynamics (with fluctuations, noise, possible chaotic behaviour,...), as in the original spirit of complex systems research. For instance, the analysis of fluctuations in network dynamics can give relevant information to distinguish how much in it is due to self-organizing, internal processes, and how much is driven by external perturbations [Argollo de Menezes and Barabasi, 2004a; 2004b]. What is important to realise it that these new approaches should not only be applied to construct descriptive models of complex dynamics (like the epidemic spreading effects in scale-free networks - [Pastor-Satorras, Vespignani, 2001]) but also to derive new general principles that throw some light on the problem of the emergence of functionality (i.e., re-organization mechanisms, like regulatory controls), hierarchies and second-order types of holism.

We consider that in order to tackle this fundamental problem further importance has to be given to bottom-up simulations of systems in between self-organization and full-fledged living or cognitive systems. Besides, such theoretical simulation models should be complemented with real experiments in the lab: real experimental data must be incorporated into the simulations, and conversely. It is crucial that in silico and in vitro approaches inform and push each other in a much more fluent, synergetic way than up to date. This is the focus, for instance, of a recent special issue on the prospects for the synthesis of 'artificial cells' [Solé, et al., 2007]. Another example of a hybrid modelling approach, coming from cognitive and neuro sciences, is given by the DARWIN robotic platform, developed by Edelman and collaborators [Krichmar and Edelman, 2005]. The control architecture of these robots, inspired by human neuroanatomical data, combines self-organized networks with modularized and hierarchically controlled processes, which all together — in continuous embodied interaction with the environment — are capable of reproducing cognitive phenomena such as categorization, associative learning, etc. Another example is Bray et al.'s model of E. coli chemotaxis integrating molecular Brownian motion and DNA molecular details to render a predictive 
model of bacterial behavior [Bray, et al., 2007].

But the point is not only methodological. The more knowledge we gain on complex systems, the stronger need we have to resort to manipulation techniques; i.e., the more dependent theoretical investigations are on the operations upon the objects of study. Traditional mechanistic methodologies in biology and cognitive science have, of course, made an intensive use of manipulation to study natural systems. However, what is new is the increasing necessity of developing experimental programs aiming at synthetic reconstructions of living or cognitive mechanisms. ${ }^{13}$ Thus, researchers are illuminated by an engineering ideal: search for the design of new genetic circuits and metabolic and neural networks. At the same time more and more scientists find that analytic de-composition needs to be complemented by synthetic re-composition (even up to the point of recomposing a different organism/neural system from deliberately modified versions of the parts). In this way, the synthetic approach is going to be increasingly used to deepen our understanding of life and mind, and to confront new problems that do not manifest through analysis. ${ }^{14}$ Therefore, the question is whether understanding life and mind, building up models of biological and cognitive organization, and the actual fabrication of living/cognitive beings are not really convergent processes.

\section{CONCLUSION: TOWARDS A NEW VIEW OF SCIENCE?}

For centuries biological and cognitive systems have been studied by analytic decomposition, trying to determine how their parts are arranged so as to generate an observed behaviour, in a similar way as the parts in human-made machines are suitably arranged to perform an externally fixed goal. 40 years ago Polanyi [1968] pointed out that the local rules harnessing physical (or chemical) laws, which would explain both the organization of living beings and machines, were complex sets of boundary conditions irreducible to general physico-chemical laws/principles. But the fundamental question remains, in so far as natural systems are concerned: where do these boundary conditions - and therefore complex organization come from? What are the main transitions in complexity that allow access to the minimal thresholds (for life and cognition)? How do these minimal forms develop and evolve without the presence and contribution of external intelligent designers?

Darwin provided a general theoretical framework that could be used to solve

\footnotetext{
${ }^{13}$ Thereby the growing interest in their origins, as well: by trying to understand the natural origins of the material mechanisms underlying biological and cognitive phenomena it will be possible to disentangle their fundamental nature, beyond descriptive accounts. This bottom-up, naturalization program is crucial not only for the future of complexity sciences, but also for the future of biology and cognitive sciences, if they are to remain proper scientific disciplines (not just technological enterprises).

${ }^{14}$ For example, synthetic biology is becoming the natural evolution of a set of procedures, like genetic engineering, required to advance in biological knowledge itself. The development of biology as a quantitative research field has simply made genetic engineering and synthetic biology unavoidable. As Rosen [1991] pointed out, the goal of understanding life is becoming more and more equivalent to that of fabricating it.
} 
this problem (or part of it). But evolutionary theory (the mechanism of natural selection) requires an initial form of organization endowed with a considerable degree of complexity [Ruiz-Mirazo, et al., 2007]. Thus, although the problem of growth and diversification of complexity could require concepts and tools coming from evolutionary theories, the problem of the origin of complex organizations cannot really be solved in that way. Modern science, in turn, has provided us with sophisticated computer techniques to perform quantitative studies of a wide variety of networks, showing emergent properties out of densely interconnected elements. Nevertheless, the sciences of complexity have not yet developed a fullfledged research program to deal with hierarchical organizations, articulated on multiple functional parts making up robust self-maintaining systems. In other words, neither evolutionary theory nor self-organization theories, as they stand today, can account for the natural origin of mechanisms.

Finding a theory that may eventually bridge this gap will require much more interdisciplinary collaboration in research. What we are learning at present is that the study of highly complex systems pushes us to overcome a strictly specialized view, constrained within a single referential framework [Pask, 1960], and to adopt a true interdisciplinary approach. The elaboration of theories and models that lead to a deeper and more global understanding of biological and cognitive systems involves the integration of very different methods and experimental data, all of them required to study even "isolated" aspects of their functioning. Experience shows that when an explanation of a complex system is achieved in terms of their more simple, lower level components, what is gained is not really more knowledge on the low-level theories but rather a new interdisciplinary theory. As it is most obviously illustrated by the research carried out on prototypical case studies or model systems (Mycoplasma, E. Coli, slime moulds, drosophila, C. elegans,...), successful explanations will be achieved through the merging of models, techniques and data coming from different studies such as genomics, development, cell physiology, psychology, neurobiology, neural networks, etc.

Actually, this is the only way to combine the network-focused, holistic perspectives with the mechanistic ones. That is why progress in the study of highly complex systems will probably imply the convergence between the holistic and the mechanistic methodologies. The understanding of complex forms of holism will progressively allow (and, at the same time, will be progressively allowed by) the merging between mechanistic explanatory methodologies, based on reductionist decomposition, and the construction of simulation models. Through these simulation models it will be possible to make explicit (and interpret under new light) processes that not only give rise to those emergent mechanisms but also assemble them into coherent and adaptive wholes.

In sum, the success of the new sciences of complexity will reframe our ways of conceiving and tackling old, open problems of science, philosophy of science and philosophy in general. As Benner and Sismour express it [2005], referring to these recent changes in the field "synthesis drives discovery and understanding in ways that analysis cannot". Biology and cognitive science seem to be at a historical 
crossroads in which the fabrication and simulation of life-like and cognitive-like systems (or subsystems) is beginning to be feasible. The consequences of this are beyond our imagination, but we are probably witnessing the first steps of a new scientific era.

\section{ACKNOWLEDGEMENTS}

Funding for this work was provided by grants 9/UPV00003.230-15840/2004 from the University of the Basque Country, IT-250-07 from the Basque Government and FFI2008-06348-C02-01 and BFU2006-01951/BMC from the Ministry of Science and Education (MICINN). K Ruiz-Mirazo holds a Ramon y Cajal research fellowship and X. Barandiaran had the support of the doctoral fellowship BFI03371AE and currently holds a post-doctoral fellowship from Ministerio de Educación y Ciencia, Programa Nacional de Movilidad de Recursos Humanos del Plan nacional de I-D+I 2008-2011. We also acknowledge Cliff Hooker's editorial suggestions for improvement and William Bechtel's helpful comments.

\section{BIBLIOGRAPHY}

[Alberch, 1982] P. Alberch. Developmental constraints in evolutionary processes. In J. T. Bonner (ed.), Evolution and Development, Dahlen Conference, Springer Verlag, New York, 1982.

[Argollo de Menezes and Barabási, 2004a] M. Argollo de Menezes and A. L. Barabási. Fluctuations in Network Dynamics. Physical Review Letters 92 (2), 028701, 2004.

[Argollo de Menezes and Barabási, 2004b] M. Argollo de Menezes and A. L. Barabási. Separating Internal and External Dynamics of Complex Systems. Physical Review Letters, 93(6), 068701, 2004.

[Barabási and Albert, 1999] A. L. Barabási, R. Albert. Emergence of scaling in random networks. Science, 286, 509-512, 1999.

[Barandiaran and Moreno, 2006a] X. Barandiaran and A. Moreno. ALife models as epistemic artefacts. In Rocha, L.M., Yaeger, L.S., Bedau, M.A., Floreano, D., Goldstone, R.L. \& Vespignani, A. (Eds.) Proceedings of the $10^{\text {th }}$ International Conference on the Simulation and Synthesis of Living Systems (ALifeX). MIT Press, Cambridge, MA,513 - 519, 2006.

[Barandiaran and Moreno, 2006b] X. Barandiaran and A. Moreno. On What Makes Certain Dynamical Systems Cognitive: A Minimally Cognitive Organization Program. Adaptive Behavior, 14(2), 171-185, 2006.

[Barandiaran and Ruiz-Mirazo, 2008] X. Barandiaran and P. Ruiz-Mirazo, eds. Modelling autonomy. BioSystems, 91(1), 2008.

[Bechtel, 2006] W. Bechtel. Discovering Cell Mechanisms. The Creation of Modern Cell Biology. Cambridge University Press, Cambridge, 2006.

[Bechtel, 2007] W. Bechtel. Mental Mechanisms: Philosophical Perspectives on Cognitive Neuroscience. Erlbaum, NJ, 2007.

[Bechtel and Abrahamsen, 2005] W. Bechtel, A. Abrahamsen. Explanation: A mechanist alternative. Studies in History and Philosophy of Biological and Biomedical Sciences, 36, 421-441, 2005.

[Beer, 1995] R. D. Beer. A dynamical systems perspective on agent-environment interaction. Artificial Intelligence, 72, 73-215, 1995.

[Benner and Sismour, 2005] S. A. Benner and A. M. Sismour. Synthetic Biology. Nat. Rev. Genet. 6, 533-542, 2005.

[Bertalanffy, 1952] L. von Bertalanffy. Problems of life: An evaluation of modern biological thought. Watts \& Co., London, 1952. 
[Block, 1980a] N. Block. What is Functionalism? In N. Block (ed.), Readings in the Philosophy of Psychology, Cambridge MA, Harvard University Press, 1980.

[Bray et al., 2007] D. Bray, L. Matthew, and K. Lipkow. The Chemotactic Behavior of Computer-Based Surrogate Bacteria. Current Biology, 17, 12-19, 2007.

[Brooks, 1991] R. A. Brooks. Intelligence without representation. Artificial Intelligence Journal, 47, 139-160, 1991.

[Brooks, 1997] R. A. Brooks. From Earwigs to Humans. Robotics and Autonomous Systems, 20(2-4), 291-304, 1997.

[Christensen, 2007] W. Christensen. The evolutionary origins of volition. In D. Spurrett, H. Kincaid, D. Ross, and L. Stephens (eds.), Distributed Cognition and the Will: Individual Volition and Social Context. MIT Press, Cambridge MA, 2007.

[Clark, 1997] A. Clark. Being there. Putting Brain, Body, and World Together Again. MIT Press, Cambridge, MA, 1997.

[Collier and Hooker, 1999] J. D. Collier and C. A. Hooker. Complexly organised dynamical systems. Open Systems and Information Dynamics, 6(3), 241-302, 1999.

[Damasio, 1994] A. R. Damasio. Descartes' Error. Emotion, Reason and the Human Brain. G. P. Putnam, New York, 1994.

[Dawkins, 1974] R. Dawkins. The Selfish Gene. Oxford University Press, Oxford, 1974.

[Edelman and Gally, 2001] G. M. Edelman and J. A. Gally. Degeneracy and complexity in biology systems. Proc. Natl. Acad. Sci. USA, 98, 13763-13768, 2001.

[Elman et al., 1996] J. L. Elman, E. Bates, M. H., Johnson, A. Karmiloff-Smith, D. Parisi, and K. Plunkett. Rethinking innateness: a connectionist perspective on development. MIT Press, Cambridge MA, 1996.

[Elsasser, 1996] W. M. Elsasser. Atom and organism. Princeton Univ. Press, Princeton NJ, 1966.

[Etxeberria et al., 2000] A. Etxeberria, A. Moreno, and J. Umerez, eds. The contribution of Artificial Life and the Sciences of Complexity to the Understanding of Autonomous Systems. CC AI:Comunication and Cognition-Artificial Intelligence, 17(3-4), 2000.

[Fodor, 1975] J. A. Fodor. The language of thought. Thomas Crowell, New York, 1975.

[Fox Keller, 2005a] E. Fox Keller. The century beyond the gene, J. Biosci., 30, 3-10, 2005.

[Fox Keller, 2005b] E. Fox Keller. Revisiting 'scale-free' networks. BioEssays, 27, 1060-1068, 2005.

[Fox Keller, 2007] E. Fox Keller. The disappearance of function from 'self-organizing systems'. In: F. Boogerd, F. Bruggeman, J-H. Hofmeyr, and H. V. Westerhoff (eds.), Systems Biology: Philosophical Foundations, Elsevier, Ámsterdam, 303-317, 2007.

[Ganti, 1987] T. Ganti. The principles of life. Oxford University Press, Oxford, 2003.

[Rev. with notes, from 1971 and OMIKK English 1987, edns., ] .

[Gell-Mann, 1994] M. Gell-Mann The quark and the jaguar. Freeman and co., New York, 1994.

[Goodwin et al., 1983] B. Goodwin, N. Holder, and C. C. Wyllie. Development and evolution. Cambridge University Press, Cambridge, 1983.

[Harold, 2001] F. Harold. The way of the cell: molecules, organisms and the order of life. Oxford_University Press, Oxford, 2001.

[Hartwell et al., 1999] L. H. Hartwell, J. J. Hopfield, S. Leibler, and A.W. Murray. From molecular to modular cell biology. Nature, 42 supp, c47-252, 1999.

[Hempel and Oppenheim, 1948] C. G. Hempel and P. Oppenheim. Studies in the logic of explanation. Philosophy of Science, 15, 137-175, 1948.

[Hopfield, 1982] J. J. Hopfield. Neural networks and physical systems with emergent collective computational abilities. Proc. Natl. Acad. Sci., 79, 2554-2558, 1982.

[Jacob, 1977] F. Jacob. Evolution as tinkering. Science, 196, 1161-1166, 1977.

[Kauffman, 1993] S. Kauffman. The origins of order: self-organization and selection in evolution. Oxford Univ. Press, Oxford, 1993.

[Kelso, 1995] J. A. S. Kelso. Dynamic patterns: The self-organization of brain and behavior. MIT Press, Cambridge MA, 1995.

[Krichmar and Edelman, 2005] J. L. Krichmar, G. M. Edelman. Brain-based devices for the study of nervous systems and the development of intelligent machines. Artificial Life, 11, $63-77,2005$

[Lewin, 1992] R. Lewin. Complexity: Life at the Edge of Chaos. MacMillan, New York, 1992 
[Laland et al., 1999] K. N. Laland, J. Odling-Smee, and M. W. Feldman. Evolutionary consequences of niche construction and their implications for ecology. Proc. Natl Acad. Sci. USA, 96(18), 10242-10247, 1999.

[Langton, 1990] C. G. Langton. Computation at the edge of chaos. Physica D, 42, 12-37, 1990.

[Lewin, 1992] R. Lewin. Complexity: life at the edge of chaos. MacMillan, New York, 1992

[Lewis, 1992] E. B. Lewis. Clusters of master control genes regulate the development of higher organisms. Journal of the American Medical Association, 267, 1524-1531, 1992.

[Lewis, 2005] M. D. Lewis. Bridging emotion theory and neurobiology through dynamic systems modeling. Behavioral and Brain Sciences, 28, 169 - 194, 2005.

[Lewontin, 2000] R. Lewontin. The triple helix. Harvard University Press, Harvard, 2000.

[Li and Vitányi, 1997] M. Li and P. Vitányi, P. An introduction to Kolmogorv complexity and its applications, Springer, New York, 1997.

[Mattick, 2004] J. S. Mattick. The hidden genetic program of complex organisms. Scientific American, *(Oct.), 61-67, 2004.

[Merleau-Ponty, 1963] M. Merleau-Ponty. The Structure of Behavior. Pittsburgh PA, Dusquene University Press, Pittsburgh PA, 1963

[Mitchell, 2006] M. Mitchell. Complex systems: Network thinking. Artificial Intelligence, $170(18), 1194-1212,2006$.

[Montoya and Solé, 2002] J. M. Montoya and R. V. Solé. Small world patterns in food webs. J. Theor. Biol., 214, 405-412, 2002.

[Morange, 2003] M. Morange. Histoire de la biologie moléculaire. La Découverte, Paris, 2003.

[Moreno and Lasa, 2003] A. Moreno and A. Lasa. From Basic Adaptivity to Early Mind. Evolution and Cognition, 9(1), 12-30, 2003.

[Newell, 1980] A. Newell. Physical Symbol Systems. Cognitive Science, 4, 135-83, 1980.

[Nicolis, 1977] G. Nicolis, I. Prigogine. Self-organization in Non-equilibrium Systems. Wiley, New York, 1977.

[Nolfi, 1997] S. Nolfi. Using emergent modularity to develop control systems for mobile robots. Adaptive Behavior, 5(3-4), 343 - 364, 1997.

[Pask, 1960] G. Pask. The Natural History of Networks. In M. C. Yovits, S. Cameron (eds.), Self-organizing Systems, Pergamon Press, Oxford, 232-263, 1960.

[Pastor-Satorras and Vespignani, 2001] R. Pastor-Satorras and A. Vespignani. Epidemic spreading in scale-free networks. Physical Review Letters, 86(14), 3200-3203, 2001.

[Pattee, 1973] H. Pattee. The Physical Basis and Origin of Hierarchical Control. In H. Pattee (ed.), Hierarchy Theory. The Challenge of Complex Systems. George Braziller, New York.

[Polanyi, 1968] M. Polanyi. Life's irreducible structure. Science, 160, 1308 - 1312, 1968.

[Port and van Gelder, 1995] R. F. Port and T. van Gelder, eds. Mind as motion: Explorations in the dynamics of cognition. The MIT Press, Cambridge MA, 1995.

[Prescott, 2007] T. J. Prescott. Forced moves or good tricks in design space? Landmarks in the evolution of neural mechanisms for action selection. Adaptive Behavior, 15(1), 9-31, 2007.

[Rachevsky, 1960] N. Rachevsky. Mathematical biophysics: Physico-mathematical foundations of biology. Dover Publications, New York, 1960.

[Ravesz et al, 2002] E. Ravesz, A. L. Somera, D. A. Mongru, Z. N, Oltvai, and A. L. Barabassi. Hierarchical Organization of Modularity in Metabolic Networks. Science, 297, 1551 — 1555, 2002 .

[Rosen, 1991] R. Rosen Life itself: A comprehensive inquiry into the nature, origin and fabrication of life. Columbia Univ. Press, New York, 1991

[Ruiz-Mirazo and Moreno, 2004] K. Ruiz-Mirazo, and A. Moreno. Basic Autonomy as a fundamental step in the synthesis of life. Artificial Life, 10(3), 235-259, 2004.

[Ruiz-Mirazo et al., 2007] K. Ruiz-Mirazo, J. Umerez, and A. Moreno. Enabling conditions for 'open-ended evolution. Biology and Philosophy, 23(1), 67-85, 2007.

[Rumelhart and McClelland, 1986] D. E. Rumelhart, J. L. McClelland, and the PDP Research Group. Parallel Distributed Processing: Explorations in the Microstructure of Cognition, vo.1: Foundations. MIT Press, Cambridge Mass., 1986.

[Schrödinger, 1944] E. Schrödinger. What is life? The physical aspect of the living cell. Cambridge Univ. Press, Cambridge, 1944.

[Skarda and Freeman, 1987] C. A. Skarda and W. J. Freeman. How brains make chaos in order to make sense of the world. Behavioral and Brain Sciences, 10, 161-195, 1987. 
[Simon, 1962] H. A. Simon The Architecture of Complexity. Proceedings of the American Philosophical Society, 106, 467-482, 1962. Reprinted in H. A. Simon, The Sciences of the Artificial, 193-229, 1969.

[Solé et al., 2002b] R. V. Solé, R. Ferrer, J. M. Montoya, and S. Valverde. Selection, Tinkering and Emergence in Complex Networks. Complexity, 8, 20-33, 2002.

[Solé et al., 2002a] R. V. Solé, I. Salazar, and J. Garcia-Fernandez. Common Pattern Formation, Modularity and Phase Transitions in a Gene Network Model of Morphogenesis. Physica A, 305, 640-647, 2002.

[Solé et al., 2007] R. V. Solé, A. Munteanu, C. Rodriguez-Caso, and J. Macía. Synthetic protocell biology: from reproduction to computation. Phil. Trans. Roy. Soc. Lond. B, 362, 1727 - 1739, 2007.

[Sporns et al., 2005] O. Sporns, G. Tononi, and R. Kotter. The human connectome: A structural description of the human brain. PLoS Comput Biol, 1(4), e42, 2005.

[Sporns et al., 2000] O. Sporns, G. Tononi, and G. M. Edelman. Theoretical neuroanatomy: relating anatomical and functional connectivity in graphs and cortical connection matrices. Cereb. Cortex, 10, 127-141, 2000.

[Steels, 1991] L. Steels Towards a theory of emergent functionality. In J. Meyer, R. Wilson (eds.), Simulation of Adaptive Behavior. MIT Press, Cambridge MA, 451-461, 1991.

[Strogatz, 2001] S. H. Strogatz Exploring complex networks. Nature, 410, 268-277, 2001.

[Thelen and Smith, 1994] E. Thelen and L. B. Smith. A dynamic systems approach to the development of cognition and action. Bradford Books/ MIT Press, Cambridge MA, 1994.

[Tononi et al., 1999] G. Tononi, O. Sporns, and G. M. Edelman. Measures of degeneracy and redundancy in biological networks. Proc. Natl. Acad. Sci. USA, 96, 3257326, 1999.

[Tsuda, 2001] I. Tsuda Toward an interpretation of dynamic neural activity in terms of chaotic dynamical systems. Behavioral and Brain Sciences, 24(5), 793-847, 2001.

[Turing, 1952] A. M. Turing The chemical basis of morphogenesis. Philos. Trans. R. Soc. Lond. B., 237, 37-72, 1952 .

[van Gulick, 1993] R. van Gulick. Who's in Charge Here? And Who's Doing All the Work? In J. Heil and A. R. Mele (eds.), Mental Causation, Clarendon Press, Oxford, 233-256, 1993.

[Waddington, 1957] C. H. Waddington. The strategy of the genes. Macmillan, London, 1957.

[Wagner and Fell, 2001] A. Wagner, D. Fell. The small world inside large metabolic networks. Proc. R. Soc. Lond. B, 268, 1803-1810, 2001.

[Waldrop, 1992] M. M. Waldrop. Complexity: The emerging science at the edge of order and chaos. Simon \& Schuster, New York, 1992

[Weaver, 1948] W. Weaver Science and Complexity. American Scientist, 36(4), 536-544, 1948.

[Reprinted in G. J. Klir. Facets of System Science, 449-456, 1991, ] .

[Weisbuch, 1986] G. Weisbuch. Networks of automata and biological organization. Journal of Theoretical Biology, 121, 255-267, 1986.

[Wolfram, 1986] S. Wolfram. Theory and applications of cellular automata. World Scientific, Singapore, 1986

[Yates, 1987] F. E. Yates (ed.). Self-organizing systems the emergence of order. Plenum Press, New York, 1987. 